\title{
Magnetospheric Multiscale Mission Attitude Dynamics: Observations from Flight Data
}

\author{
Trevor Williams ${ }^{1}$, Seth Shulman², Joseph Sedlak ${ }^{3}$, \\ Neil Ottenstein ${ }^{3}$, Brian Lounsbury ${ }^{2}$
}

\author{
AIAA SPACE 2016 \\ Long Beach, CA \\ Sept. 16, 2016
}

${ }^{1}$ NASA Goddard Space Flight Center; ${ }^{2}$ Honeywell Tech Solutions, Inc.; ${ }^{3}$ ai Solutions, Inc. 


\section{Summary of Presentation}

- MMS: four helioscience spacecraft flying in formation

- Spinners (3.05 RPM); 60 m wires

- Thrusters for attitude, orbit control

- Star camera attitude sensors

- Summary of presentation:

- Spin axis targeting

- Effects of environmental torques

- Effects of active potential control device (jets of Indium ions) on observed spacecraft spin rate

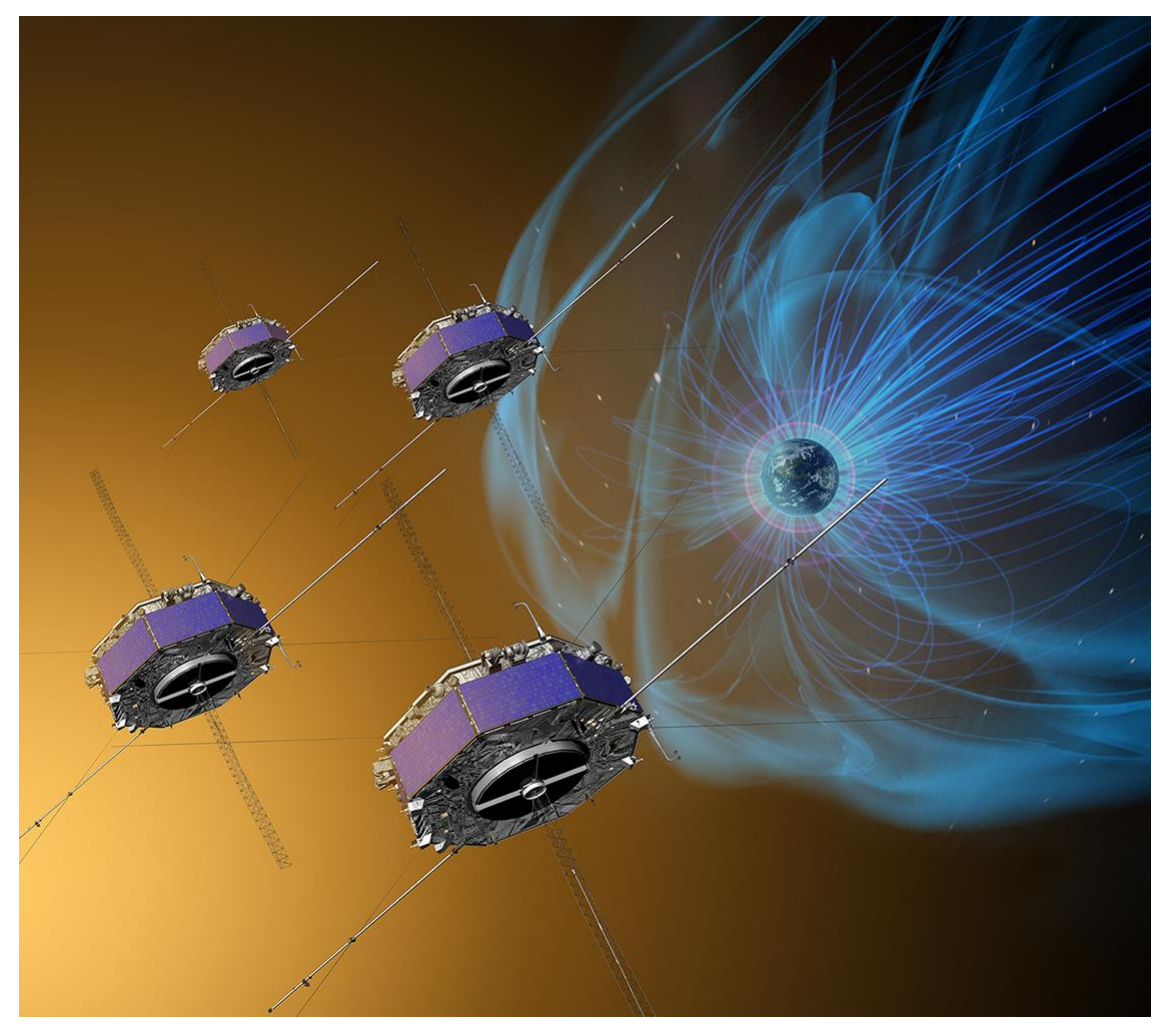

- Derivation of effective thrust

- Analysis of MMS4 impact event in Feb. 2016, using attitude data 


\section{Spin Axis Target}

- Spin axis (body Z-axis) must be near ecliptic pole

- This attitude ensures sunlight does not fall on upper deck

- Upper deck illumination would cause emission of photoelectrons that would perturb the local plasma and field measurements

- However, spin axis needs some tilt towards the Sun

- Tilt prevents shadows from pre-amplifiers on wire booms from crossing the spherical detectors at ends of wire booms

- Shadows cause momentary interruption of photo-emissive electron cloud around detector spheres, again perturbing field measurements

- Target box for science ops is isosceles trapezoid, roughly $2.5 \mathrm{deg} \times 2.5 \mathrm{deg}$ with center tipped $3.5 \mathrm{deg}$ toward Sun 


\section{Environmental Torques}

- MMS Attitude Ground System (AGS) predicts when spin axis will drift to the edge of the target box

- AGS plans attitude slews to center or to opposite edge of box to maximize time between maneuvers

- Spin axis drift depends on seasonally changing environmental torques

- Very rough order-of-magnitude estimates of torques

- Gravity-gradient: $\quad 10^{-4} \mathrm{~N}-\mathrm{m}$

- Solar pressure: $10^{-6} \mathrm{~N}-\mathrm{m}$

- Aerodynamic drag: $10^{-7} \mathrm{~N}-\mathrm{m}$

- So, only gravity-gradient (GG) torque is used in AGS predictions 


\section{Predicted Precession of Spin Axis}

- AGS predicts GG drift of the spin axis direction

- Early mission, after all booms deployed, drift was 0.05 deg per orbit (orbital period was close to 24 hours)

- Plot shows accumulated drift error for 35 days with no maneuvers

- Error in drift prediction was approximately 0.00034 deg per orbit

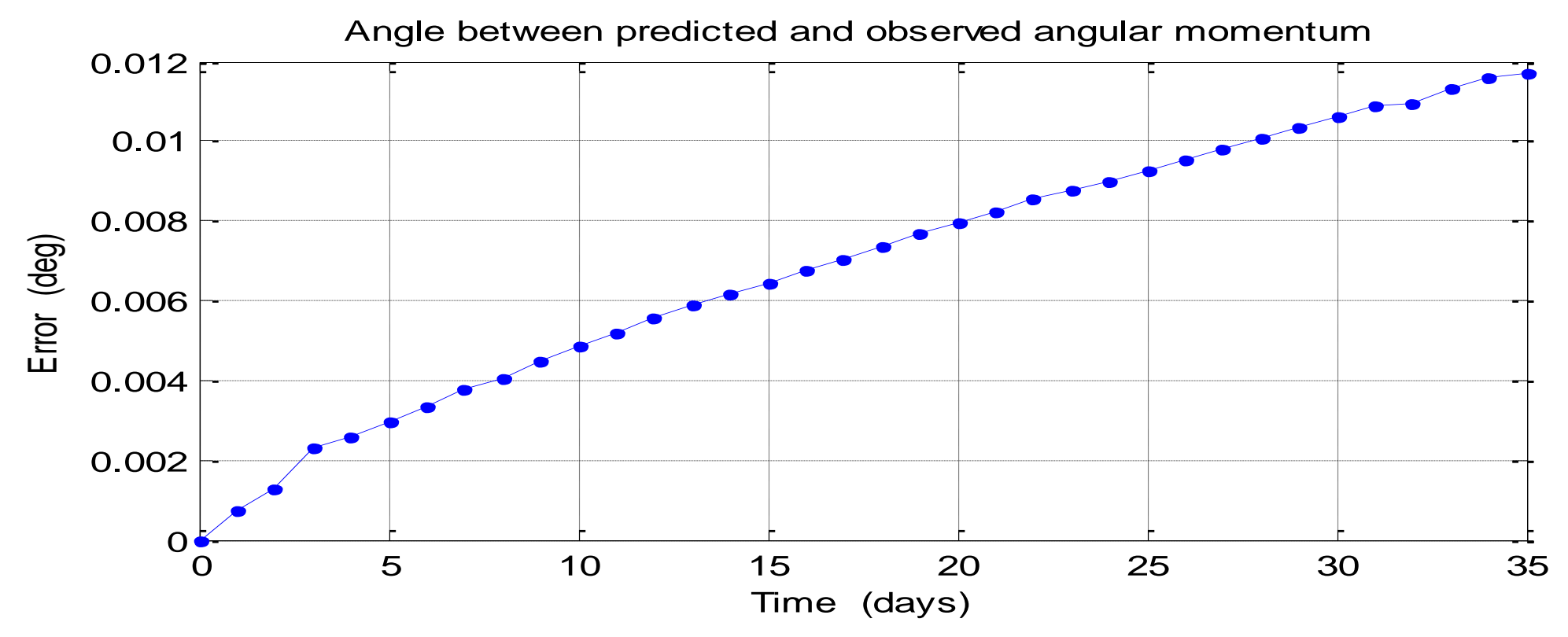




\section{Seasonal Variation of Precession}

- Magnitude and direction of GG precession vary seasonally

- Orbit normal drifted approx. 21 deg during one year, affecting GG torque

- Target box center follows the Sun motion of one deg per day

- Attitude maneuvers are performed every 2 to 4 weeks to stay in target box

- Plots show seasonal variation of magnitude of precession per orbit and angle between direction of precession and motion of box center

- GG precession is helping when angle is near zero (i.e., longer time between maneuvers), but GG magnitude is smallest then (so it doesn't help much)

- Avg. time between maneuvers was 30 days for the months when angle was small, and was 22.5 days for the entire post-commissioning time span
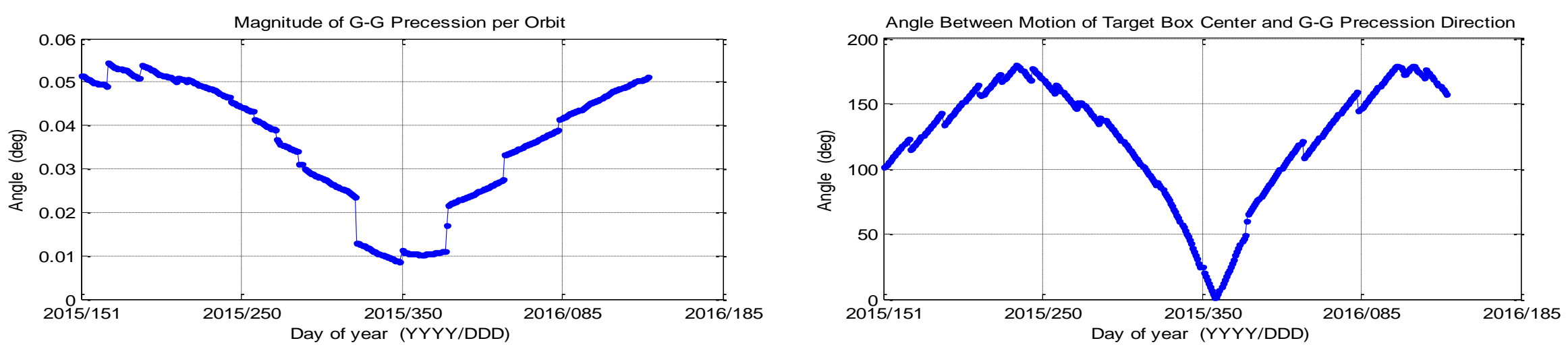


\section{Observed Change In Spin Rate}

- Distinct spin rate change observed at ASPOC (Active Spacecraft Potential Control Investigation) turn on and duty cycle changes

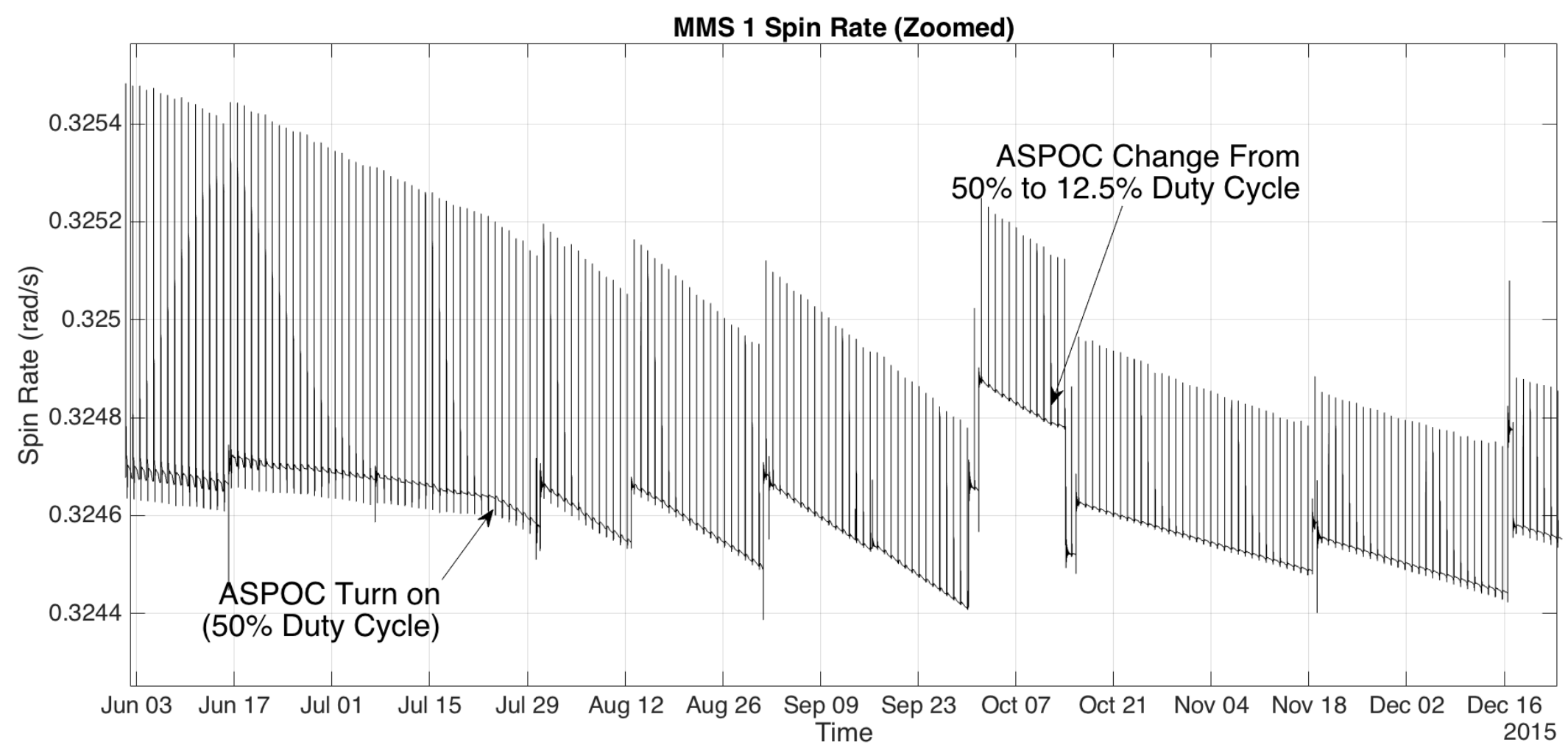




\section{ASPOC Characteristics}

- Purpose is to neutralize buildup of positive floating potential produced by the spacecraft/environment interaction

- Strong potential created between emitter and extractor

- Indium atoms ionized and accelerated by this electric field
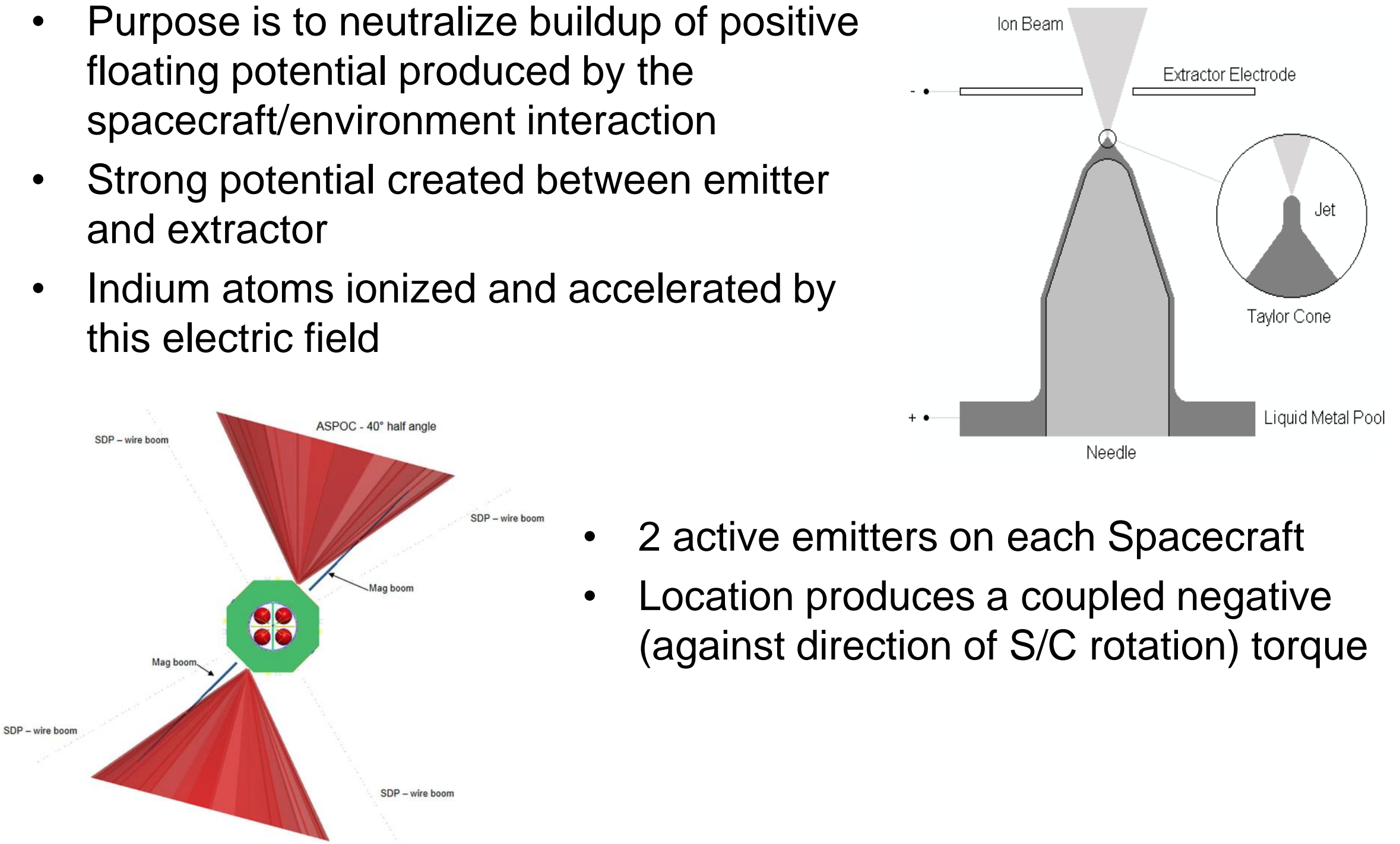

- 2 active emitters on each Spacecraft

- Location produces a coupled negative (against direction of $S / C$ rotation) torque 


\section{Determining Empirical Thrust}

- Time between maneuvers defined as a sample

- Using average deceleration, center of mass, moment of inertia, and emitter energy an empirical emitter thrust is calculated

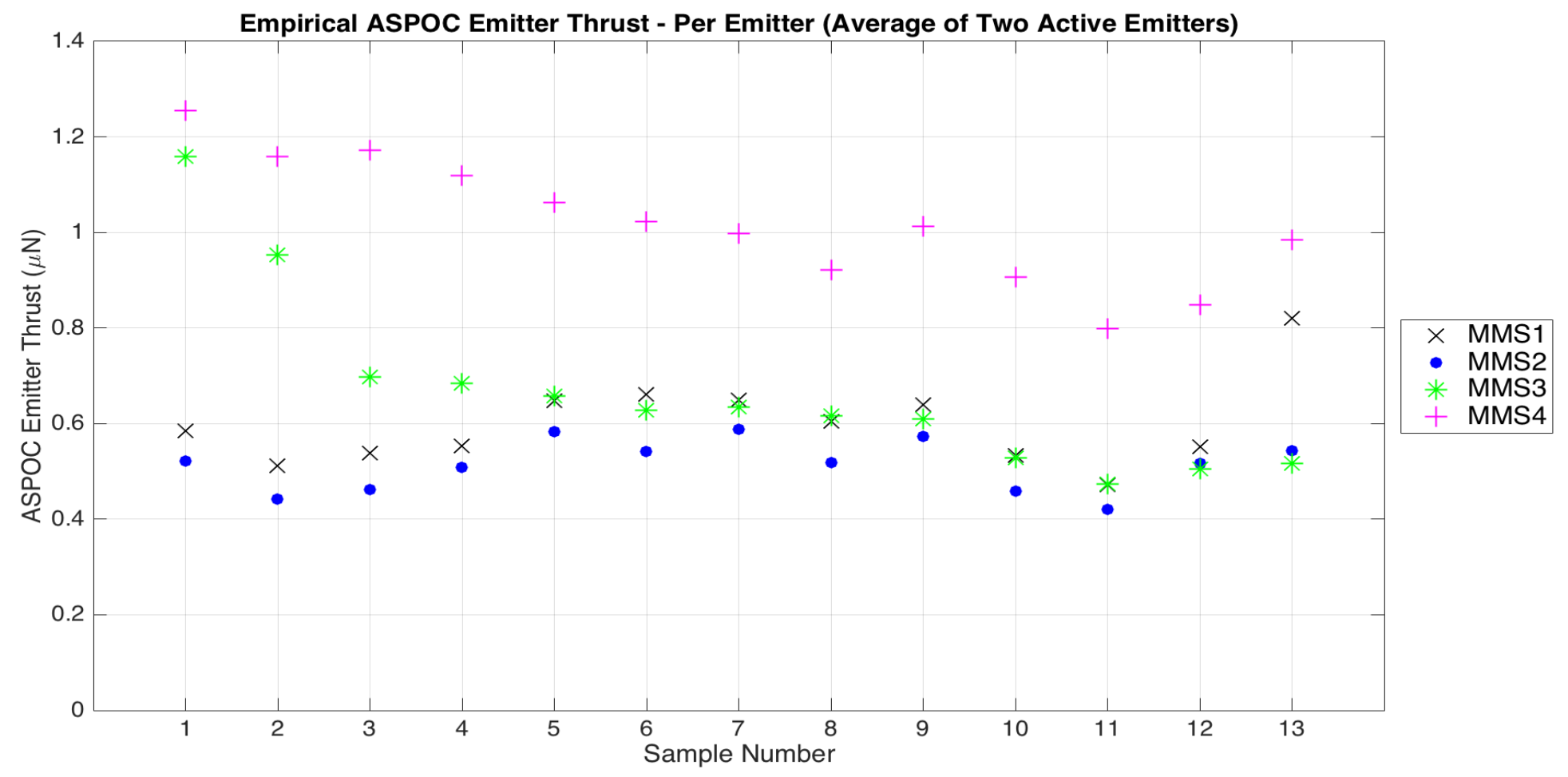




\section{Determining Empirical Thrust}

- Time between maneuvers defined as a sample

- Using average deceleration, center of mass, moment of inertia, and emitter energy an empirical emitter thrust is calculated

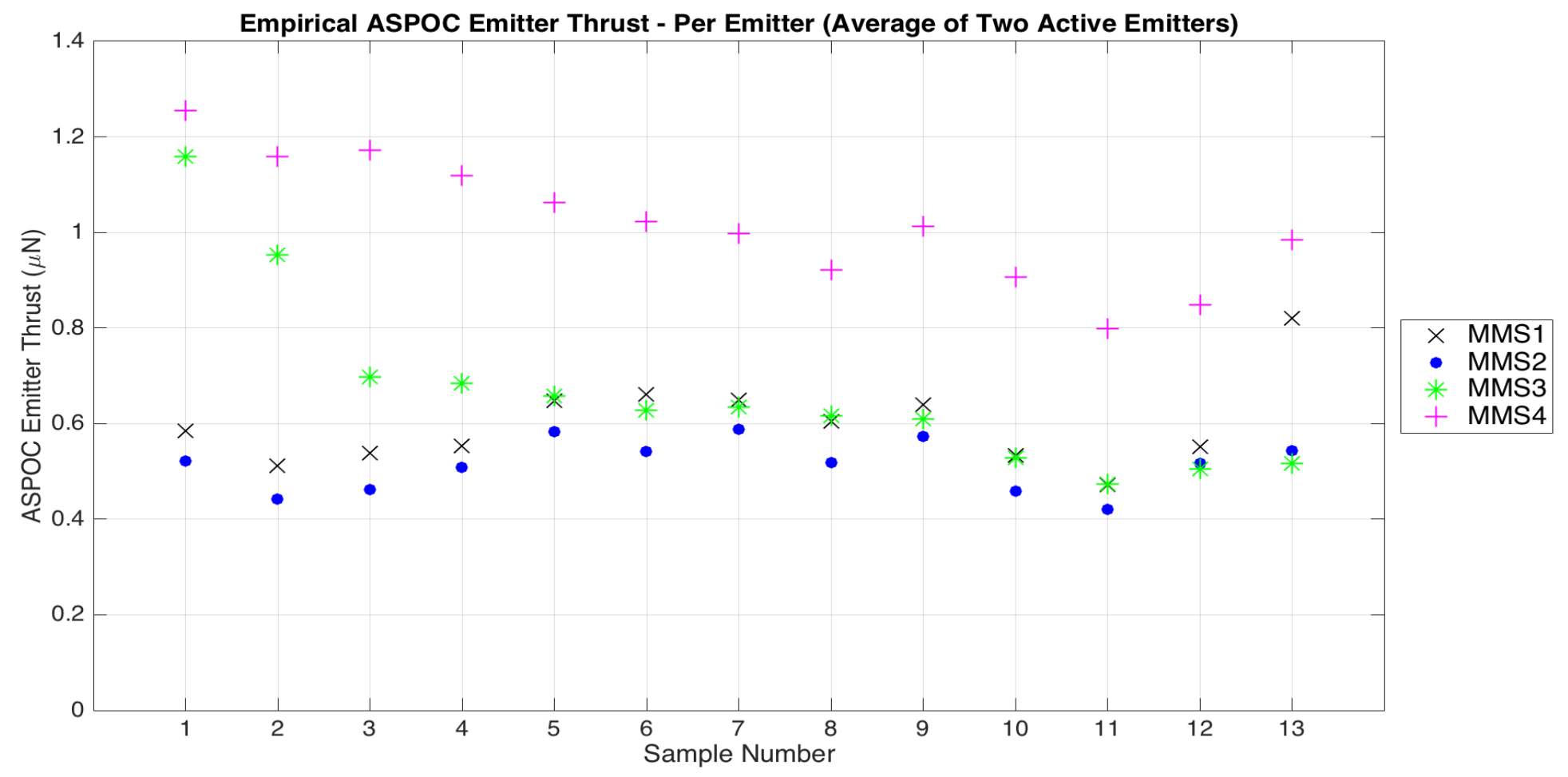




\section{Summary of MMS4 Impact Event}

- MMS4 relevant data observations:

- Failure of one shunt resistor

- Accelerometers detected spacecraft disturbance

- Star cameras "blinded" by non-star objects; reset by fault detection

- Small attitude excursions (change in spin axis direction; nutation etc.)

- Science instruments detected plasma around spacecraft

- MMS4 state at event:

- Radius 48,176 km (7.553 $\left.R_{E}\right): 6,012 \mathrm{~km}$ greater than GEO radius

- Latitude -21.2 deg: 17,403 km below equatorial GEO plane

- $\quad 4,414 \mathrm{~km}$ below Ecliptic

- Orbital speed $2.661 \mathrm{~km} / \mathrm{s}$

- Geometry of event:

- Impact, possibly oblique, on bottom face of spacecraft

- Goals of analysis: to the (limited) accuracy possible with given data

- Identify candidate impactor sources

- Estimate likely approach direction

- Estimate likely relative speed and mass of impactor

Sept. 16,20 Frstimate likely kinetic energy of initial impact 


\section{Impact Location (Shunt Resistor)}
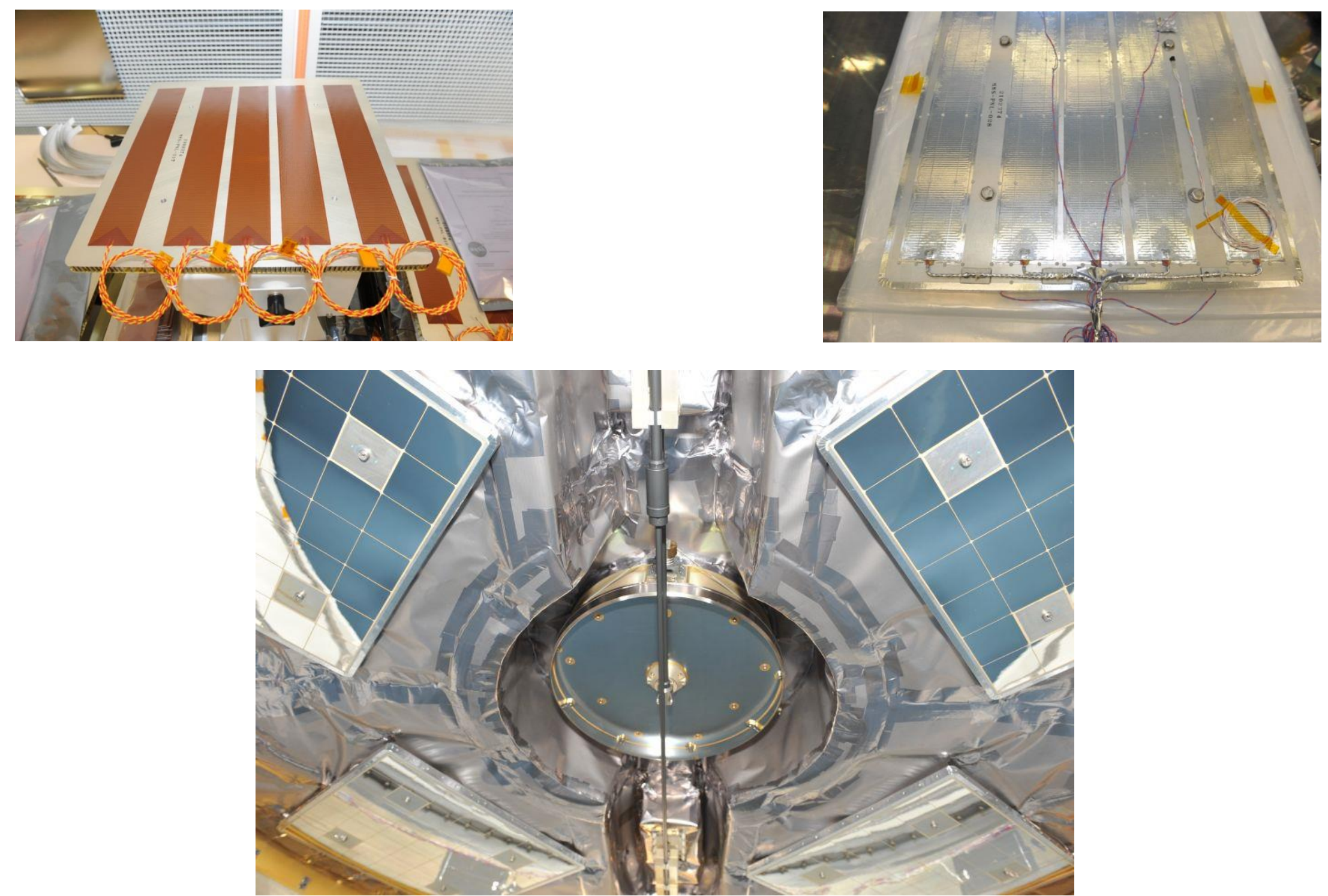

Sept. 16, 2016 


\section{Shunt Resistor Data}

. . .

MMS-4: EPS - MOSFET Shunt Current Max Drop (ITPS Computed MAX every hour)

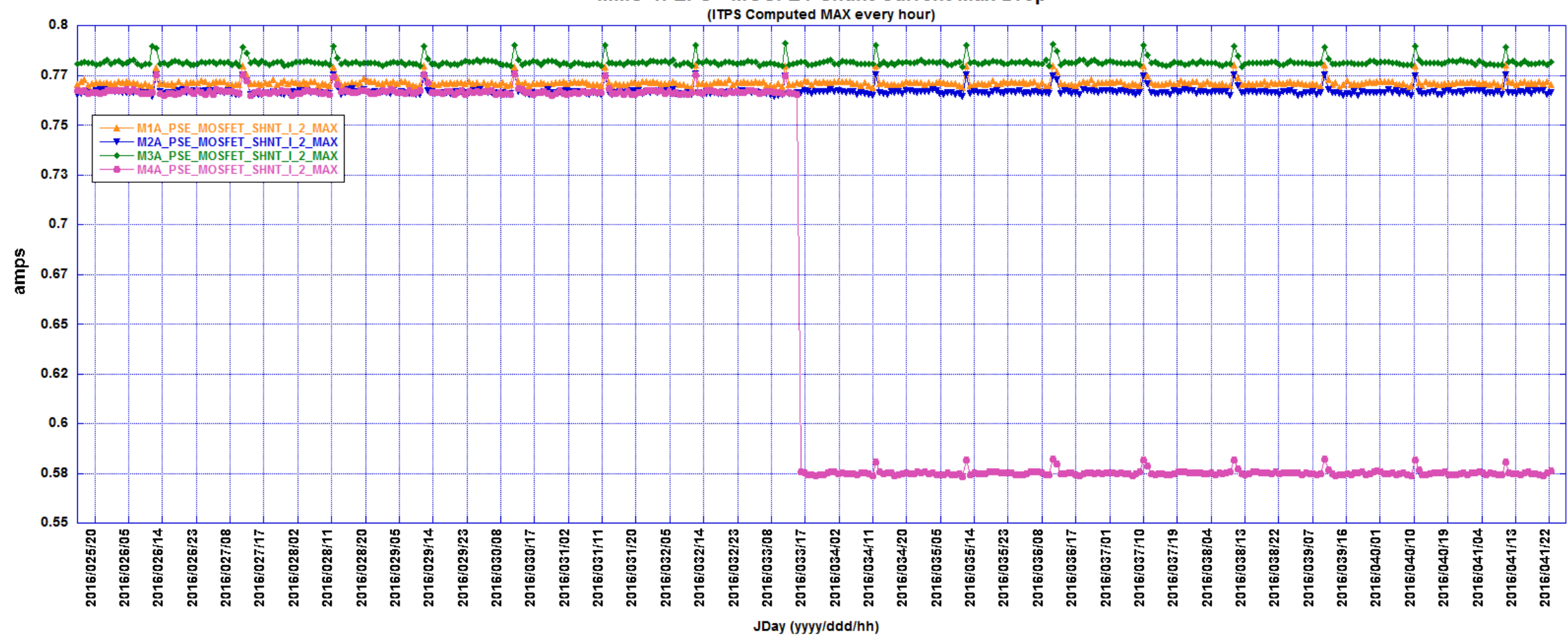




\section{Analysis Methodology}

- Use relative sizes of initial spikes in accelerometer signals caused by event to estimate velocity direction of impactor relative to MMS

- Use change in MMS spin axis direction produced by event, together with known spacecraft angular momentum, to derive the transverse angular momentum applied to MMS by impactor

- From known impact point on spacecraft and estimated approach direction, this allows the linear momentum $\left(\mathrm{mv}_{\text {rel }}\right)$ of impactor relative to MMS CM to be computed

- From known position on orbit of impact, the MMS orbital velocity at the time of the event is known

- For assumed impactor population, can hence find estimated speed of impactor relative to MMS

- From the known linear momentum $m v_{\text {rel }}$ and relative speed $v_{\text {rel }}$, we can then estimate the mass $m$ of the impactor

- Use these to estimate kinetic energy of initial impact, $\mathrm{T}=0.5 \mathrm{mv}_{\text {rel }}{ }^{2}$ 


\section{Accelerometer Measurements}

$X$-axis: Initial spike -0.8 micro-g

Note: All three axes only sampled every $30 \mathrm{~s}$, so actual first motion may not be observed

Y-axis: Initial spike 2.8 micro-g
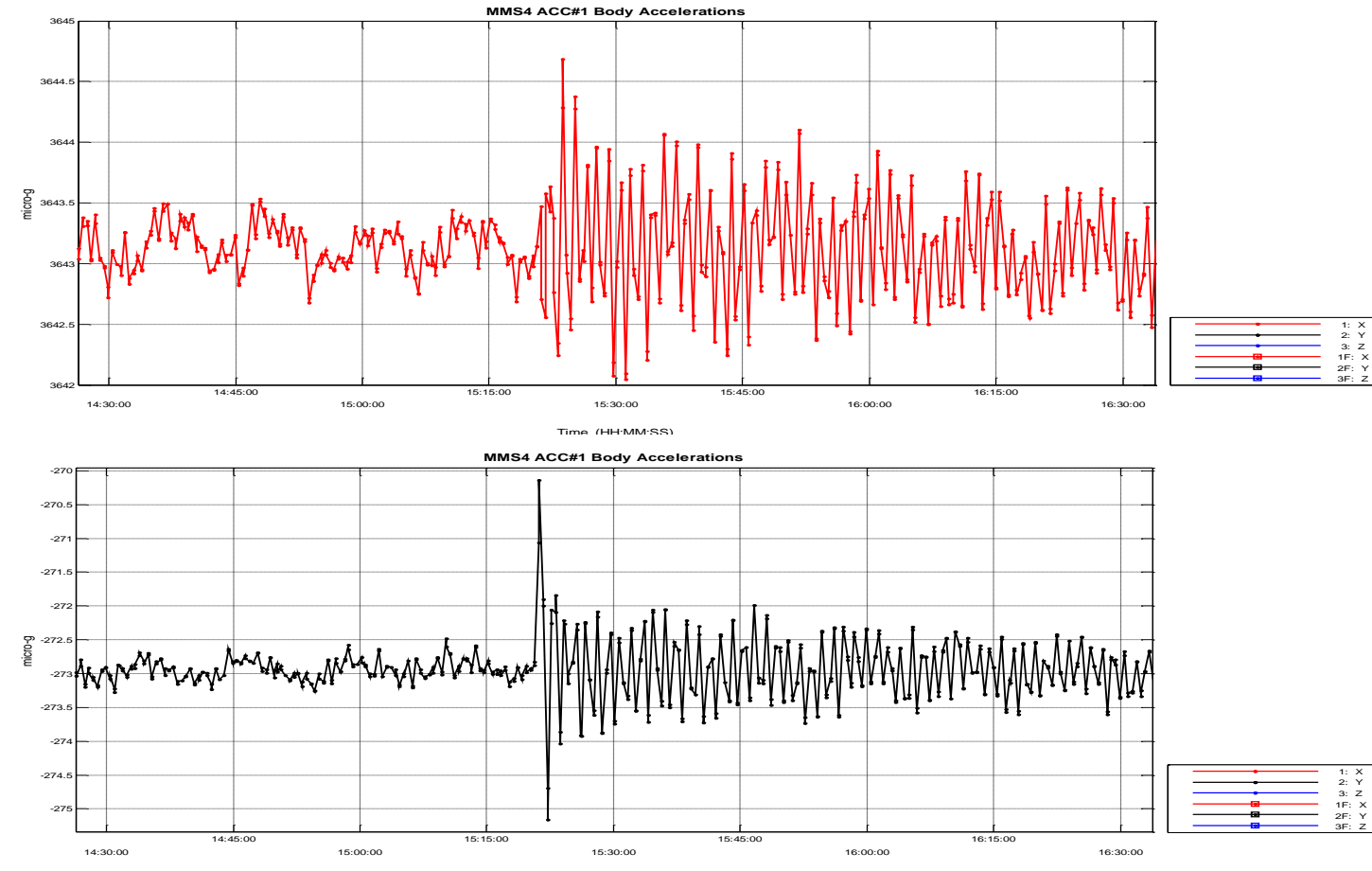

Z-axis: Initial spike -1.7 micro-g

Resulting relative velocity direction estimate: 30.3 deg below spin plane

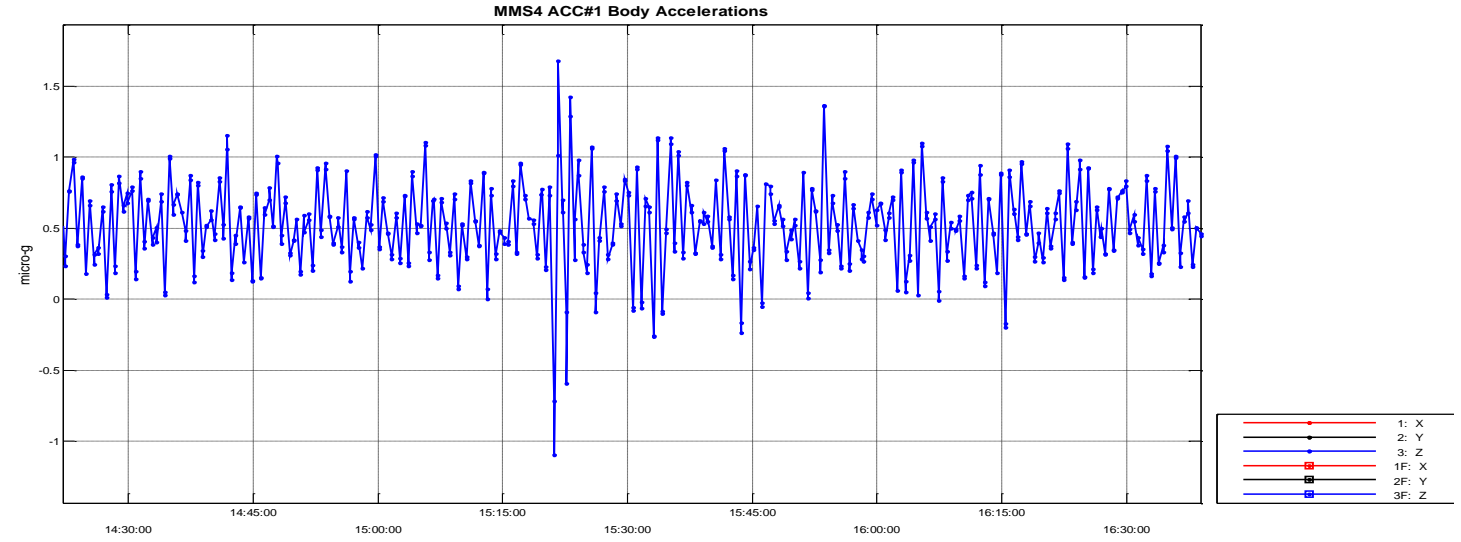




\section{Rotation Rates, Transverse and Axial}

Transverse:

Nutation/boom

vibration evident

Note brief dropout resulting from star cameras being blinded/resetting

Axial: No change in spin rate evident

Sept. 16, 2016
MMS4 Rotation Rates in Body Frame
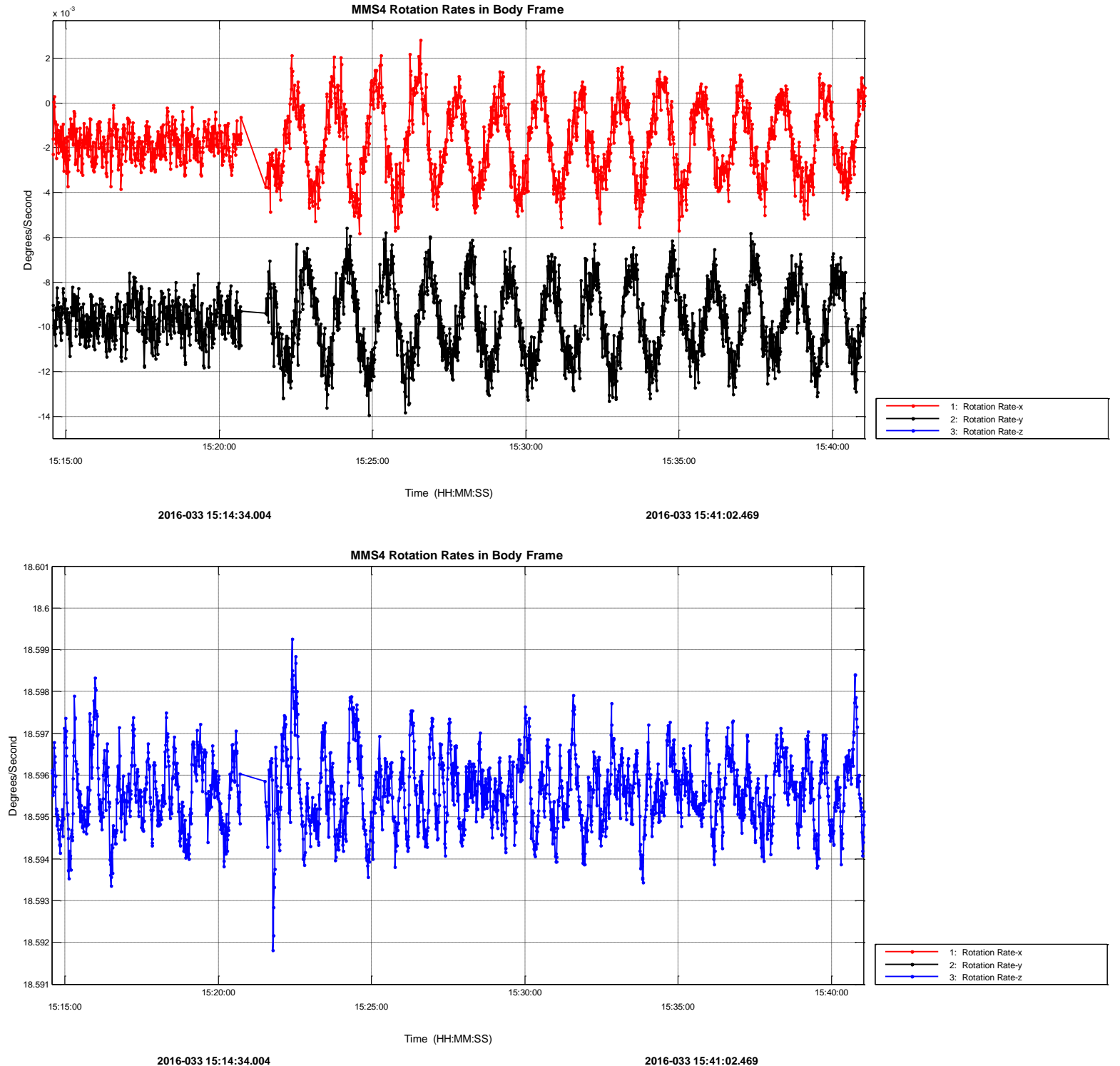


\section{Pointing Angle Before Event}

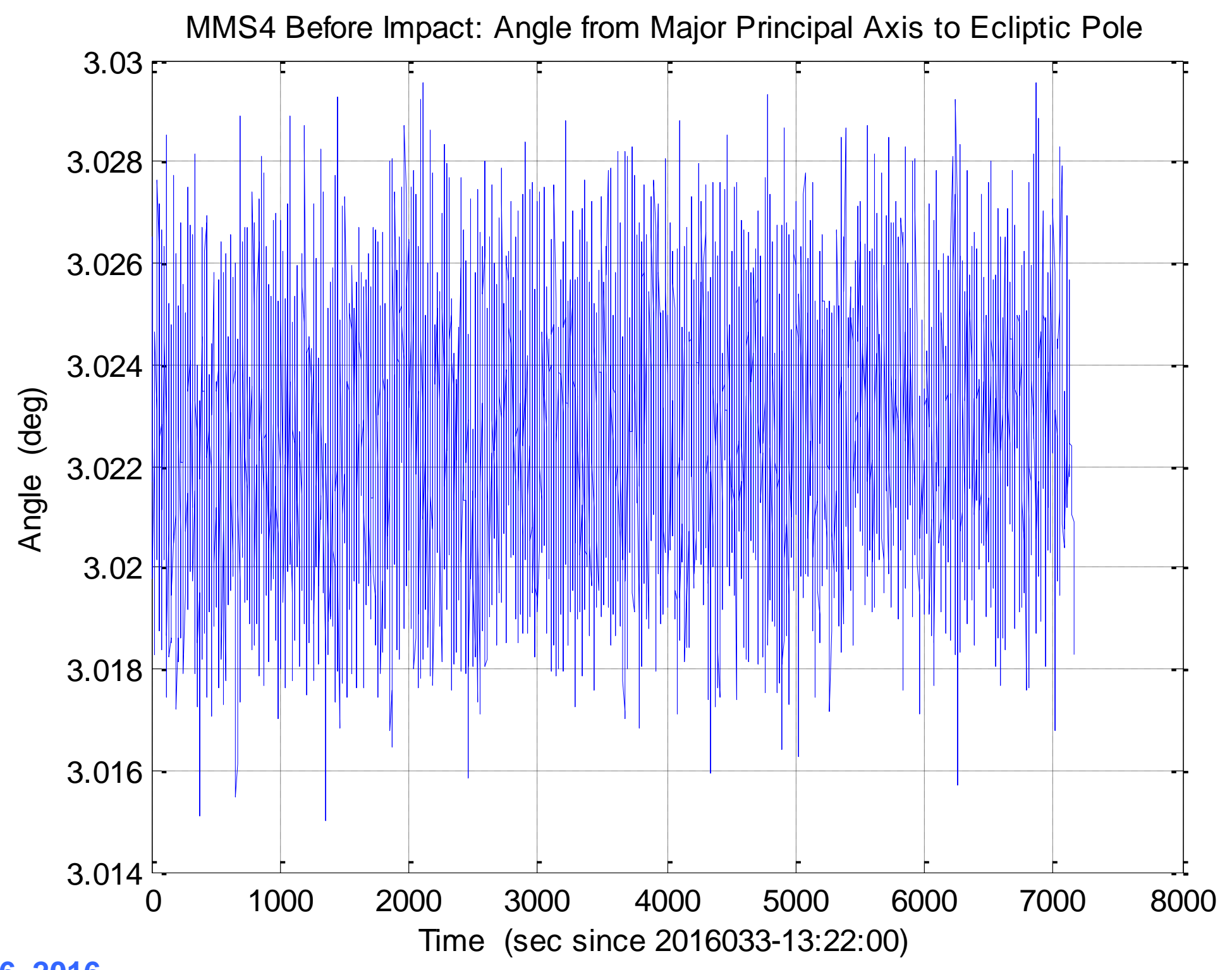

Sept. 16, 2016 


\section{FFT of Pointing Angle Before Event}

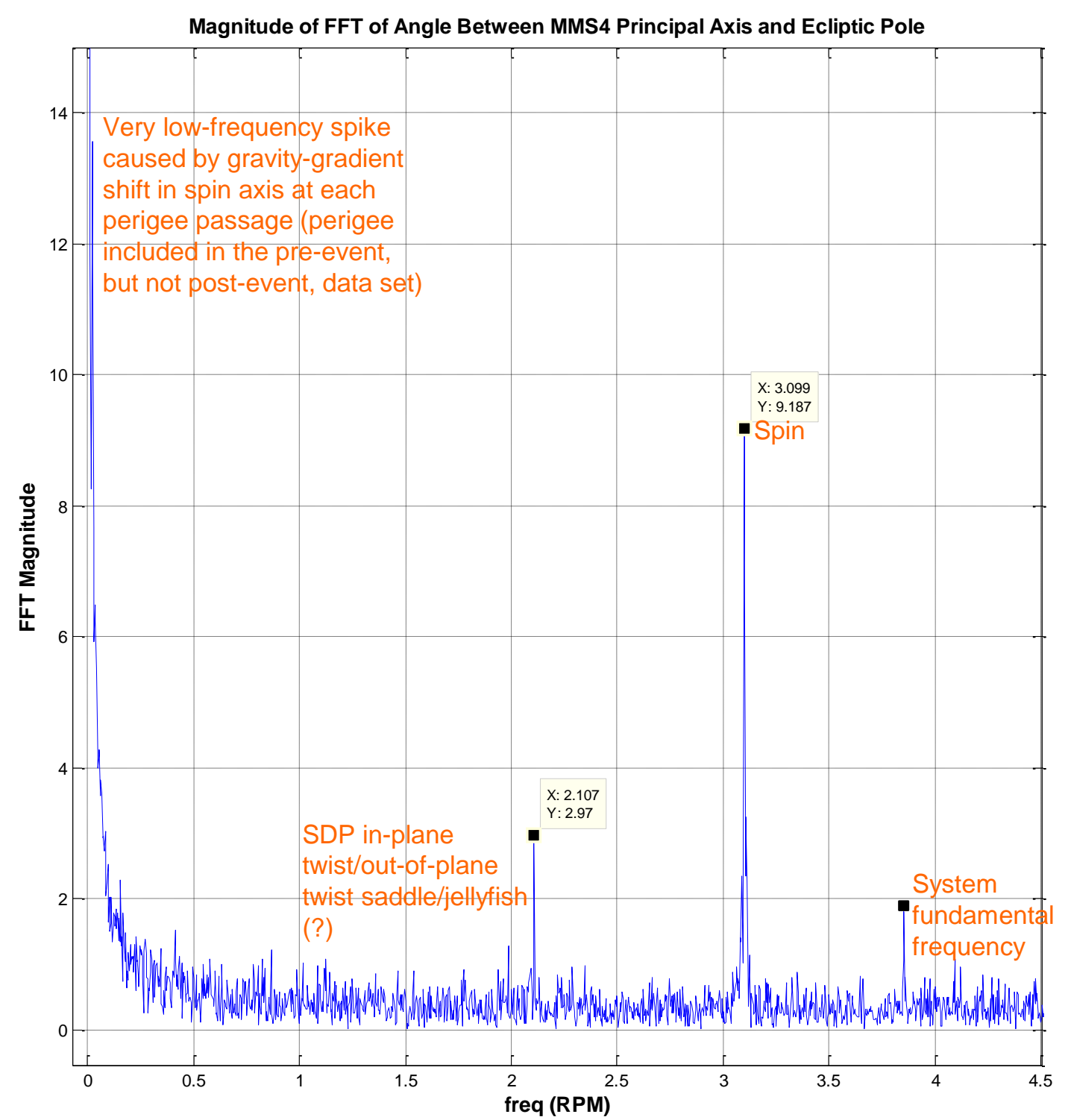

Sept. 16, 2016 


\section{Pointing Angle After Event}

Vibration with period of $\sim 400 \mathrm{~s}$ dominates response

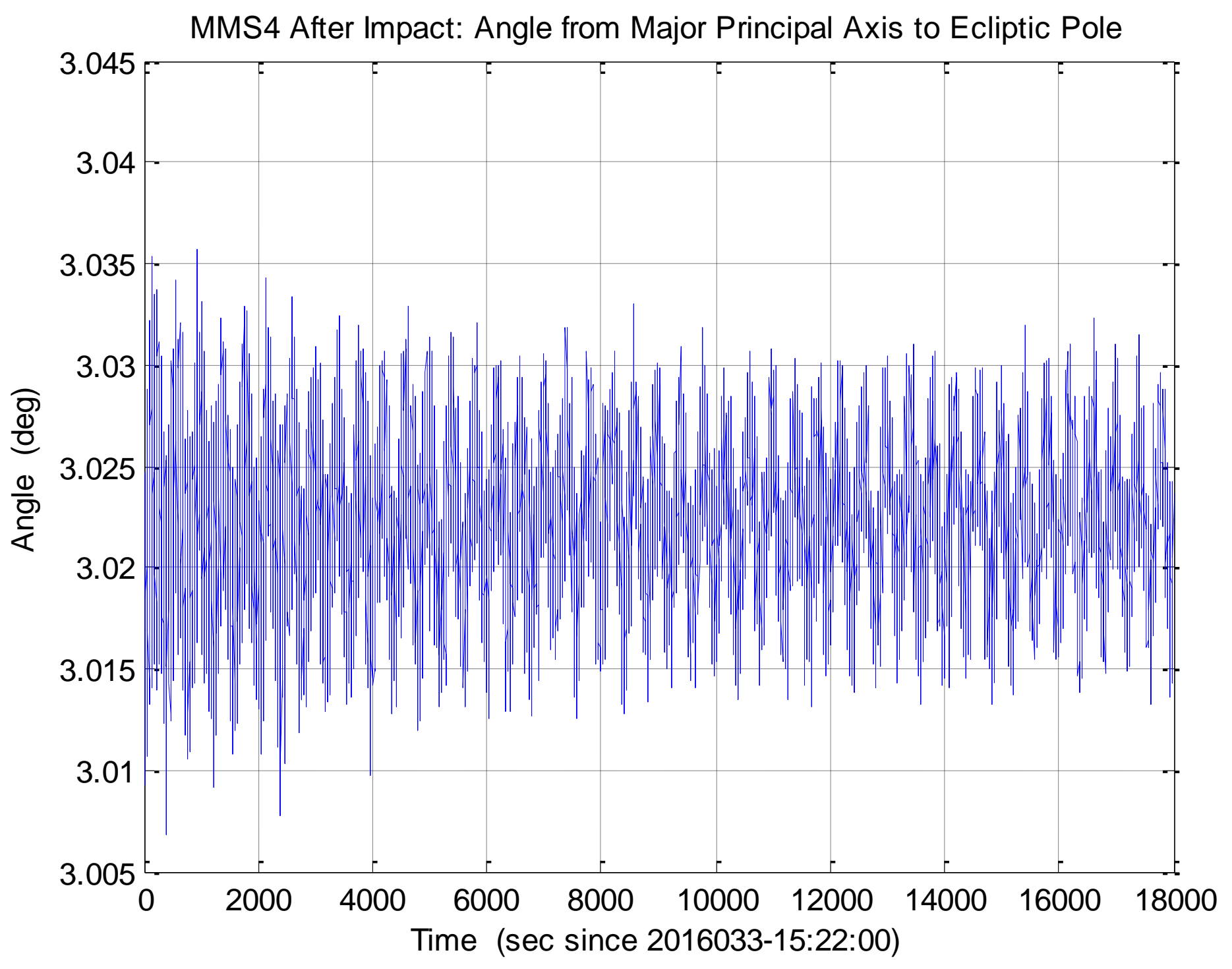




\section{FFT of Pointing Angle After Event}

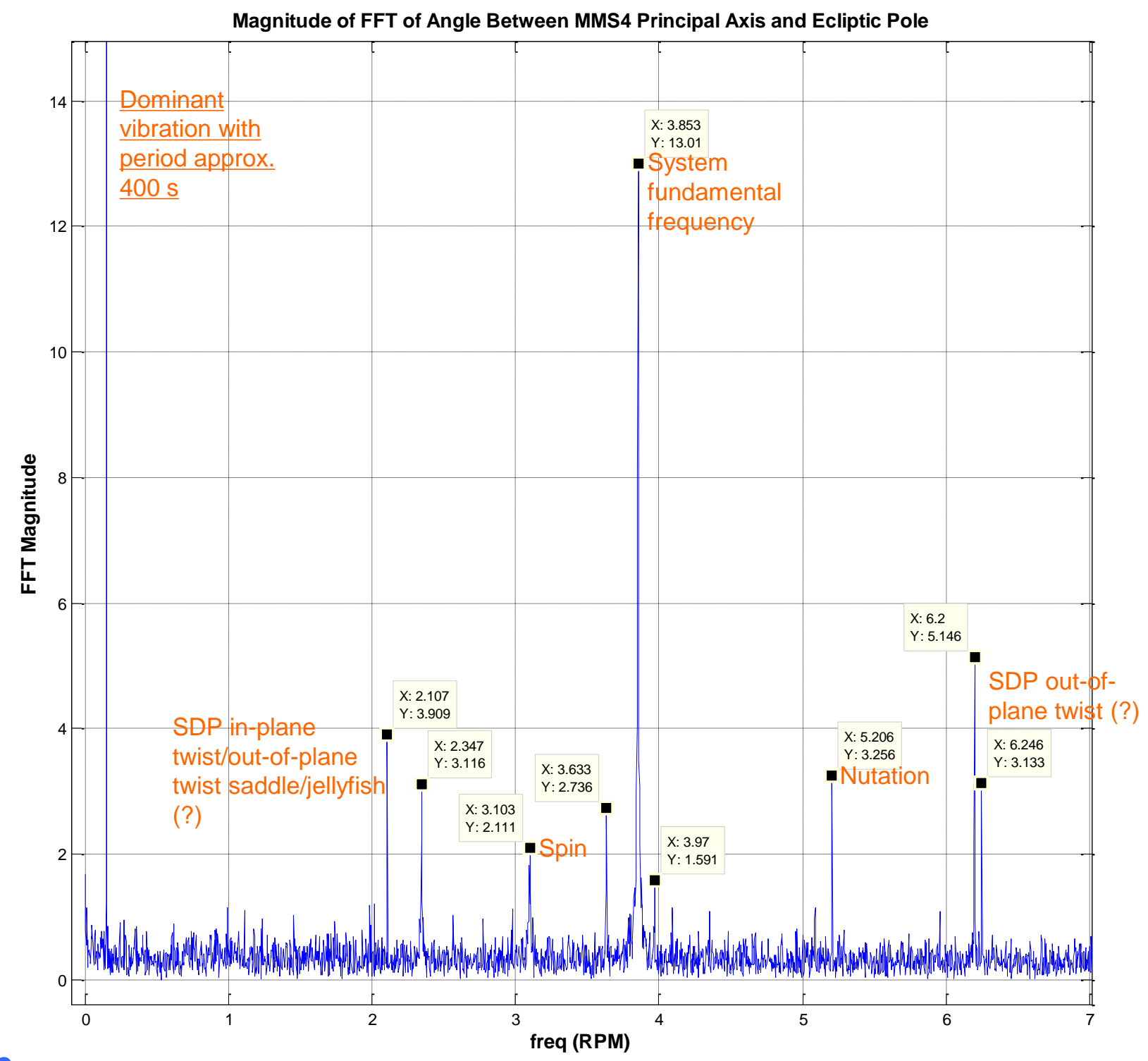

Sept. 16, 2016 


\section{Possible Sources of Impactor - 1}

- Two possible sources have been studied:

- Micrometeoroid (dust particle)

- Debris originating in GEO and perturbed by lunisolar gravitation plus solar radiation pressure (SRP) to point of impact

- Micrometeoroid (dust) population:

- Overall mass range: 10-14 to $10^{0} \mathrm{gm}$

- Peak mass range: $\sim 10^{-8}$ to $10^{-3} \mathrm{gm}\left(\sim 2 \times 10^{-4}-0.9 \mathrm{~mm}\right.$ diameter)

- Flux tails off quickly: $\sim 10^{-3}$ as high at $1 \mathrm{~mm}$ diameter as at $0.1 \mathrm{~mm}^{*}$
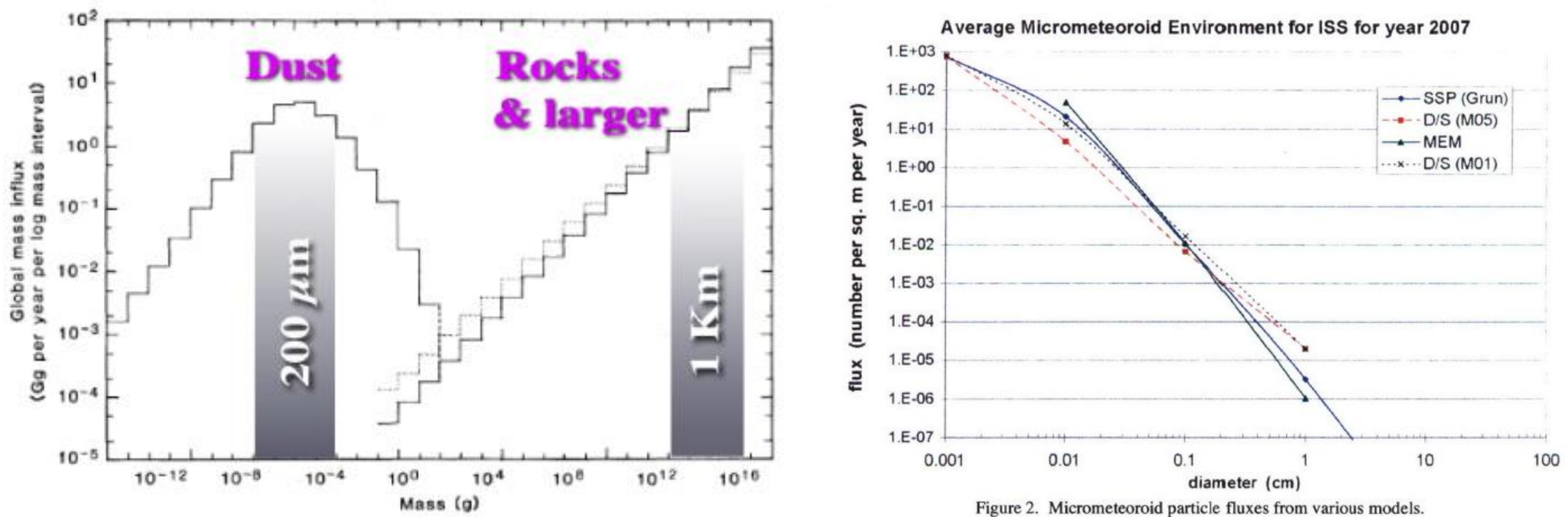

* Fig. 2, "Micrometeoroid and Orbital Debris Environments for the International Space Station", Peterson and Lynch, 2008 


\section{Possible Sources of Impactor - 2}

- Debris originating in GEO: GEO spacecraft have inclinations that oscillate between 0 and $\sim 15$ deg, as a result of lunisolar perturbations. The impact latitude of -21.2 deg exceeds this range; the impact radius was also $6,012 \mathrm{~km}$ above GEO

- However, objects released from GEO that have high area/mass ratios (> 15 $\mathrm{m}^{2} / \mathrm{kg}$ ) experience significant solar radiation pressure (SRP) perturbations in eccentricity (and so radius) and inclination

- References:

- "Long-Term Dynamics of High Area-to-Mass Ratio Objects in High Earth Orbit", Rosengren and Scheeres, 2013

- "Long-Term Evolution of Geosynchronous Orbital Debris with High Area-to-Mass Ratios", Pardini and Anselmo, 2006

- Possible debris source: multi-layer insulation (MLI). MLI degrades in GEO. See Tedlar thin film before, after 3 years simulated GEO*:
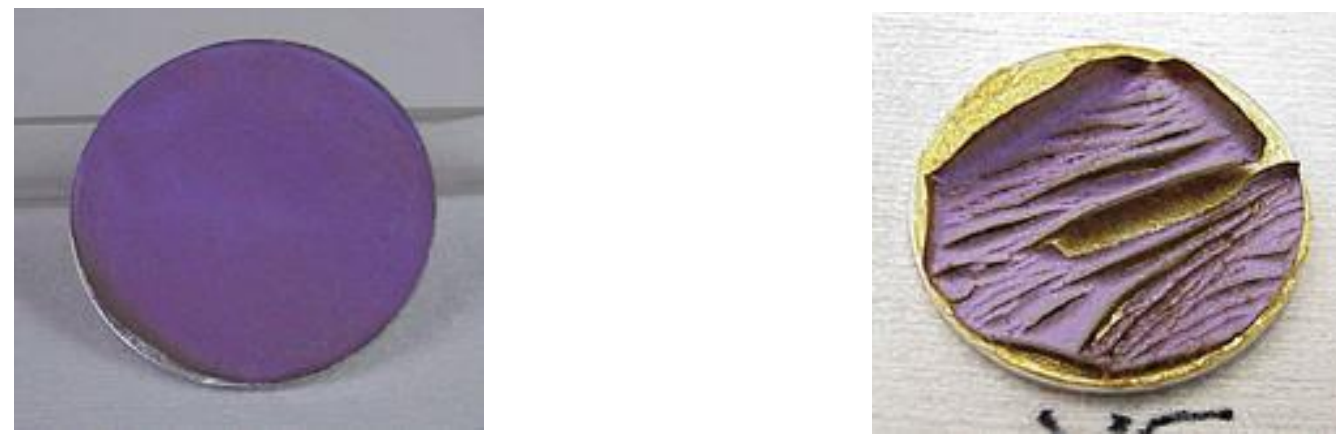

- Representative MLI layer density $40 \mathrm{gm} / \mathrm{m}^{2}$; area/mass $25 \mathrm{~m}^{2} / \mathrm{kg}$ 


\section{Particle Mass, Kinetic Energy Estimates}

- Linear momentum of impactor must produce observed change in spin axis direction of $0.00157 \mathrm{deg}$

- Mass, KE estimates differ for the two candidate particle sources, as a result of the different relative speeds between particle and MMS4

- Micrometeoroid:

- "Typical" relative speed $15 \mathrm{~km} / \mathrm{s}$ (very wide variation is possible)

- Resulting estimated particle mass $8.48 \times 10^{-3} \mathrm{gm}$

- Resulting kinetic energy $953.9 \mathrm{~J}$ (46.6\% of muzzle energy of AK-47)

- Debris of GEO origin:

- Orbital speed of debris at impact $2.661 \mathrm{~km} / \mathrm{s}$

- Resulting relative speed $\sim 4.292 \mathrm{~km} / \mathrm{s}$ (depends on geometry)

- Resulting estimated debris mass $2.96 \times 10^{-2} \mathrm{gm}$

- If from an MLI layer with representative density $40 \mathrm{gm} / \mathrm{m}^{2}$, this yields an area of $7.41 \times 10^{-4} \mathrm{~m}^{2}$, e.g. a square $2.72 \mathrm{~cm}$ on a side

- Resulting kinetic energy $272.9 \mathrm{~J}$ (13.3\% of muzzle energy of AK-47)

- From this analysis, it is difficult to select between the candidates. Perhaps impact dynamics analysis can lead to a determination 


\section{Backup Material}




\section{Angular Momentum}

Transverse:

Nutation/boom vibration evident

Axial: No change in spin rate evident. Consistent with shunt location being close to spin axis
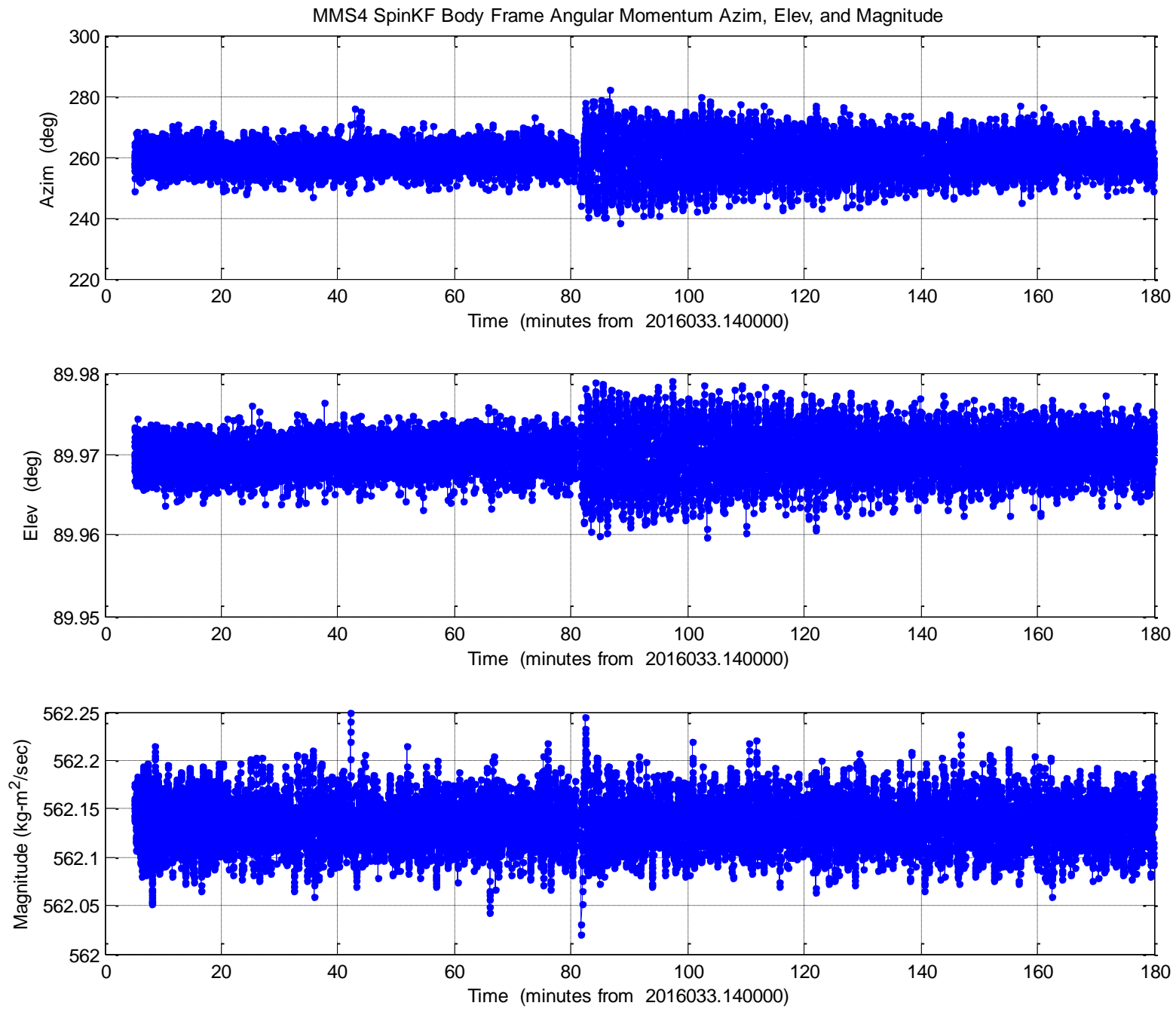


\section{Pointing Angle After Previous Maneuver NASA}

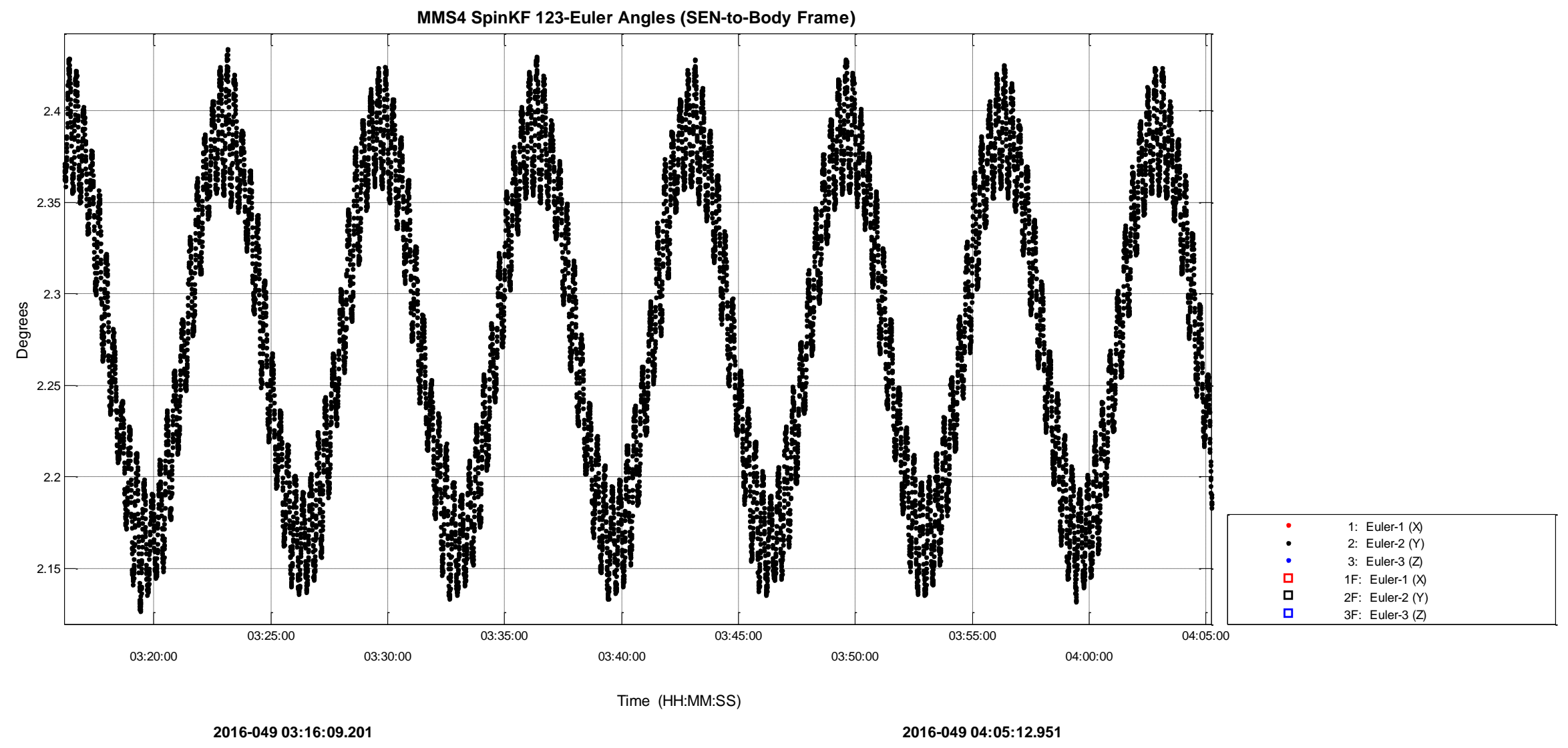

- Oscillation at same $400 \mathrm{~s}$ period is clearly visible

- Observed after all spacecraft maneuvers

- Must be wire boom dynamics excited by thrusting/impact acceleration of central spacecraft body 